\title{
Philympics 2021: Prophage Predictions Perplex Programs
}

\section{[version 1; peer review: 1 approved with reservations, 1 not}

\section{approved]}

\author{
Michael J. Roach (D1), Katelyn McNair2, Sarah K Giles', Laura K Inglis', Evan Pargin1, \\ Simon Roux ${ }^{3}$, Przemysław Decewicz ${ }^{4}$, Robert A. Edwards ${ }^{1}$
}

${ }^{1}$ Flinders Accelerator for Microbiome Exploration, Flinders University, Adelaide, SA, 5042, Australia

${ }^{2}$ Computational Sciences Research Center, San Diego State University, San Diego, CA, 92182, USA

${ }^{3}$ DOE Joint Genome Institute, Lawrence Berkeley National Laboratory, Berkeley, CA, 94720, USA

${ }^{4}$ Department of Environmental Microbiology and Biotechnology, Institute of Microbiology, Faculty of Biology, University of Warsaw, Warsaw, 02-096, Poland

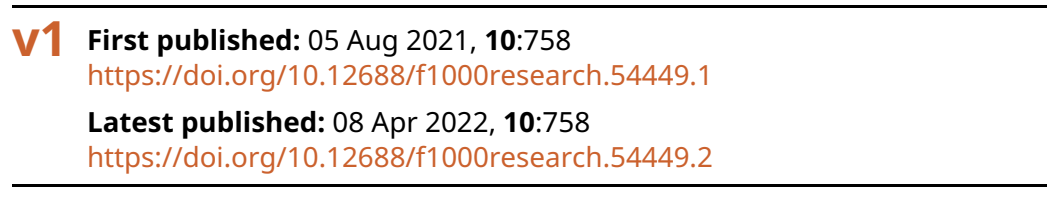

\section{Abstract}

\section{Background}

Most bacterial genomes contain integrated

bacteriophages-prophages-in various states of decay. Many are active and able to excise from the genome and replicate, while others are cryptic prophages, remnants of their former selves. Over the last two decades, many computational tools have been developed to identify the prophage components of bacterial genomes, and it is a particularly active area for the application of machine learning approaches. However, progress is hindered and comparisons thwarted because there are no manually curated bacterial genomes that can be used to test new prophage prediction algorithms.

Methods

We present a library of gold-standard bacterial genome annotations that include manually curated prophage annotations, and a computational framework to compare the predictions from different algorithms. We use this suite to compare all extant stand-alone prophage prediction algorithms to identify their strengths and weaknesses. We provide a FAIR dataset for prophage identification, and demonstrate the accuracy, precision, recall, and $f_{1}$ score from the analysis of seven different algorithms for the prediction of prophages. Results

We identified different strengths and weaknesses between the prophage prediction tools. Several tools exhibit exceptional $f_{1}$ scores, while others have better recall at the expense of more false positives. The tools vary greatly in runtime performance with few exhibiting all desirable qualities for large-scale analyses.

Conclusions

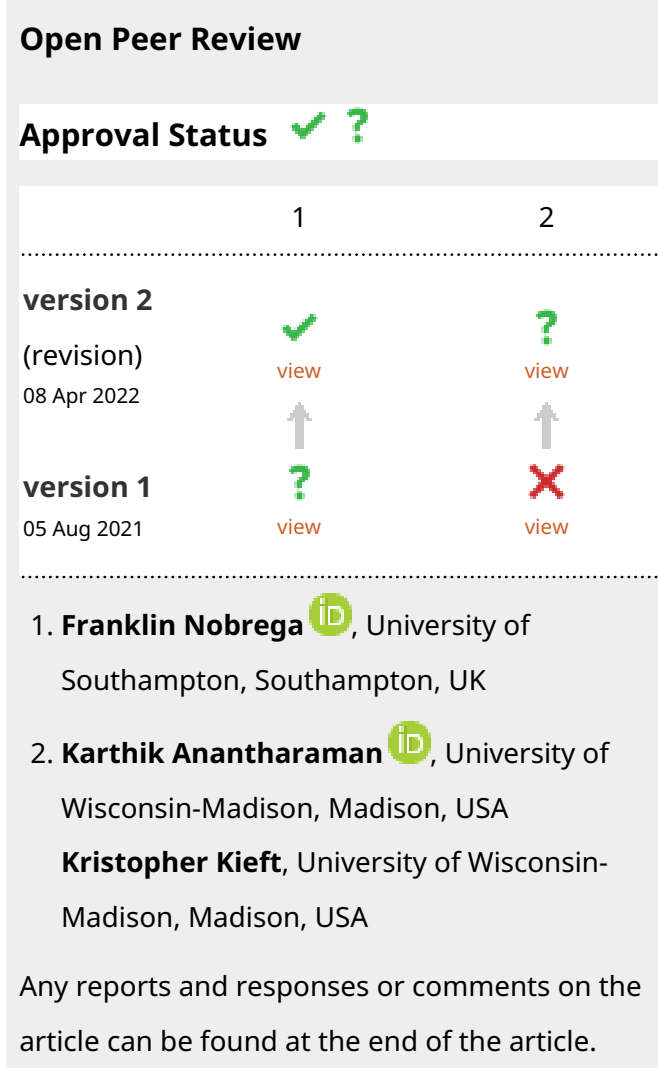


Our library of gold-standard prophage annotations and benchmarking framework provide a valuable resource for exploring strengths and weaknesses of current and future prophage annotation tools. We discuss caveats and concerns in this analysis, how those concerns may be mitigated, and avenues for future improvements. This framework will help developers identify opportunities for improvement and test updates. It will also help users in determining the tools that are best suited for their analysis.

Keywords software comparison, bioinformatics tool, lysogen genome, temperate phage, prokaryotic virus

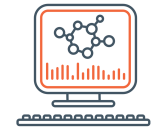

This article is included in the Bioinformatics

gateway.

Corresponding author: Michael J. Roach (michael.roach@flinders.edu.au)

Author roles: Roach MJ: Data Curation, Formal Analysis, Investigation, Methodology, Software, Validation, Visualization, Writing Original Draft Preparation, Writing - Review \& Editing; McNair K: Data Curation, Investigation, Methodology, Writing - Review \& Editing; Giles SK: Data Curation, Investigation, Writing - Review \& Editing; Inglis LK: Data Curation, Investigation, Writing - Review \& Editing; Pargin E: Data Curation, Investigation, Writing - Review \& Editing; Roux S: Investigation, Software, Writing - Review \& Editing; Decewicz P: Data Curation, Investigation, Methodology, Resources, Software, Supervision, Writing - Review \& Editing; Edwards RA:

Conceptualization, Data Curation, Formal Analysis, Funding Acquisition, Methodology, Project Administration, Resources, Software, Supervision, Visualization, Writing - Original Draft Preparation, Writing - Review \& Editing

Competing interests: No competing interests were disclosed.

Grant information: This work supported by the National Institute Of Diabetes And Digestive And Kidney Diseases of the National Institutes of Health under Award Number RC2DK116713 to RAE. The support provided by Flinders University for HPC research resources is acknowledged.

The funders had no role in study design, data collection and analysis, decision to publish, or preparation of the manuscript.

Copyright: @ 2021 Roach MJ et al. This is an open access article distributed under the terms of the Creative Commons Attribution License, which permits unrestricted use, distribution, and reproduction in any medium, provided the original work is properly cited.

How to cite this article: Roach MJ, McNair K, Giles SK et al. Philympics 2021: Prophage Predictions Perplex Programs [version 1; peer review: 1 approved with reservations, 1 not approved] F1000Research 2021, 10:758 https://doi.org/10.12688/f1000research.54449.1

First published: 05 Aug 2021, 10:758 https://doi.org/10.12688/f1000research.54449.1 


\section{Introduction}

Bacteriophages (phages), viruses that infect bacteria, can be either temperate or virulent. Temperate phages may integrate into their bacterial host genome and the host-integrated phage genome is referred to as a prophage. Prophages are ubiquitous and may constitute as much as 20 percent of bacterial genomes (Casjens, 2003). Prophages replicate as part of the host bacterial genomes until external conditions trigger a transition into the virulent lytic cycle, resulting in replication and packaging of phages and typically the death of the host bacteria. Prophages generally contain a set of core genes with a conserved gene order that facilitate integration into the host genome, assembly of phage structural components, replication, and lysis of the host cell (Kang et al., 2017; Canchaya et al., 2003). As well as these core genes, phages can contain an array of accessory metabolic genes that can effect significant phenotypic changes in the host bacteria (Breitbart, 2012). For instance, many prophages encode virulence factors such as toxins, or they can encode fitness factors such as nutrient uptake systems (Brüssow et al., 2004). Lastly, most prophages encode a variety of super-infection exclusion mechanisms to prevent concurrent phage infections, including restriction/modification systems, toxin/antitoxin genes, repressors, etc. (Calendar, 1988). The function of most prophage accessory genes remains unknown.

Core (pro) phage genes have long been used for identifying prophage regions. However, there are other unique characteristics that can distinguish prophages from their host genomes: bacterial genomes have a GC skew that correlates with direction of replication, and the insertion of prophages will generally disrupt this GC bias (Grigoriev, 1998). Transcript direction (Campbell, 2002) and length of prophage proteins have also proven to be useful metrics in predicting prophages (Akhter et al., 2012; Song et al., 2019), where phage genes are generally smaller and are oriented in the same direction (Dutilh et al., 2014). Likewise, gene density tends to be higher in phage genomes and intergenic space shorter (Amgarten et al., 2018; McNair et al., 2019).

Over the last two decades many prophage prediction tools have been developed, and they fall into two broad classes: (1) web-based tools where users upload a bacterial genome and retrieve annotations including PHASTER (Arndt et al., 2016), Prophage Hunter (Song et al., 2019), Prophinder (Lima-Mendez et al., 2008), PhageWeb (Sousa et al., 2018), and RAST (Aziz et al., 2008); and (2) command-line tools where users download a program and database to run the predictions locally (although some of these also provide a web interface for remote execution). In this work we focus on this latter set of tools (Table 1) because web-based tools typically do not handle the large numbers of simultaneous requests required to run comparisons across many genomes.

Despite the abundance of prophage prediction algorithms, there has never been either a set of reference genomes against which all tools can be compared, nor a unified framework for comparing those tools to identify their relative strengths and weaknesses or to identify opportunities for improvement. We generated a set of manually annotated bacterial genomes released under the FAIR principles (Findable, Accessible, Interoperable, and Reusable), and developed an openly available and accessible framework to compare prophage prediction tools.

\section{Methods}

Running the tools

To assess the accuracy of the different prophage prediction tools, a set of 49 gold-standard publicly available bacterial genomes with manually curated prophage annotations was generated. The genomes and prophage annotations currently included are available in Tables S1 and S2. The genomes are in GenBank format and file conversion scripts are included in the framework to convert those files to formats used by the different software. The tools that are currently included in the framework are outlined in Table 1. Snakemake (Köster \& Rahmann, 2012) pipelines utilising conda (Anaconda Software Distribution. Conda. v4.10.1, April 2021 (Conda, RRID:SCR_018317)) package manager environments were created for each tool to handle the installation of the tool and its dependencies, running of the analyses, output file conversion to a standardized format, and benchmarking of the run stage. Where possible, annotations from the GenBank files were used in the analysis to promote consistency between comparisons. Additional pipelines were created for running PhiSpy using the included training sets for the appropriate genera, and for running PhiSpy with pVOG (Grazziotin et al., 2017) HMMs and these are also available in the repository. DBSCAN-SWA was not able to consistently finish when using GenBank files as input, and instead the genome files in fasta format were used. Another pipeline was created to pool the results from each tool and some comparisons are illustrated in the included Jupyter notebook. Testing and development of the pipelines were conducted on Flinders University's DeepThought HPC infrastructure. The final benchmarking analysis was performed on a stand-alone node consisting of dual Intel ${ }^{\circledR}$ Xeon $^{\circledR}$ Gold 6242R processors (40 cores, 80 threads), 768 GB of RAM, and 58 TB of disk space. Each tool was executed on all genomes in parallel (one thread per job), with no other jobs running.

\section{Benchmark metrics}

There are many potential ways to compare prophage predictions: For instance, is it more important to capture all prophage regions or minimise false positives? Is it more important to identify all the phage-encoded genes, or the 


\begin{tabular}{|c|c|c|c|c|c|c|}
\hline Tool (year) & Version & $\begin{array}{l}\text { Package } \\
\text { manager }\end{array}$ & Dependencies & $\begin{array}{l}\text { Database } \\
\text { size }\end{array}$ & Approach & Citation \\
\hline $\begin{array}{l}\text { Phage } \\
\text { Finder } \\
\text { (2006) }\end{array}$ & 2.1 & & $\begin{array}{l}\text { Aragorn, blast- } \\
\text { legacy, hmmer, } \\
\text { infernal, mummer, } \\
\text { trnascan-se }\end{array}$ & $93 \mathrm{MB}$ & $\begin{array}{l}\text { Legacy- } \\
\text { BLAST, } \\
\text { HMMs }\end{array}$ & (Fouts, 2006) \\
\hline $\begin{array}{l}\text { PhiSpy } \\
(2012)\end{array}$ & 4.2 .6 & $\begin{array}{l}\text { conda, } \\
\text { pip }\end{array}$ & $\begin{array}{l}\text { Python3, biopython, } \\
\text { numpy, scipy }\end{array}$ & $\begin{array}{l}47 \mathrm{MB} \\
\text { required, } \\
733 \mathrm{MB} \\
\text { optional } \\
\text { (pVOGs) }\end{array}$ & $\begin{array}{l}\text { Gene and } \\
\text { nucleotide } \\
\text { metrics, } \\
\text { AT/CG skew, } \\
\text { kmer } \\
\text { comparison, } \\
\text { machine } \\
\text { learning, } \\
\text { HMMs, } \\
\text { annotations }\end{array}$ & $\begin{array}{l}\text { (Akhter } \\
\text { et al., 2012) }\end{array}$ \\
\hline $\begin{array}{l}\text { VirSorter } \\
\text { (2015) }\end{array}$ & 1.0.6 & conda & $\begin{array}{l}\text { mcl, muscle, blast+, } \\
\text { bioperl, hmmer, } \\
\text { diamond, } \\
\text { metagene_annotator }\end{array}$ & $13 \mathrm{~GB}$ & $\begin{array}{l}\text { Alignments, } \\
\text { HMMs }\end{array}$ & $\begin{array}{l}\text { (Roux } \\
\text { et al., 2015) }\end{array}$ \\
\hline $\begin{array}{l}\text { Phigaro } \\
(2020)\end{array}$ & 2.3 .0 & $\begin{array}{l}\text { conda, } \\
\text { pip }\end{array}$ & $\begin{array}{l}\text { Python3, } \\
\text { beautifulsoup4, } \\
\text { biopython, bs4, } \\
\text { hmmer, Ixml, numpy, } \\
\text { pandas, plotly, } \\
\text { prodigal, pyyaml, } \\
\text { shsix }\end{array}$ & $1.6 \mathrm{~GB}$ & HMMs & $\begin{array}{l}\text { (Starikova } \\
\text { et al., 2020) }\end{array}$ \\
\hline $\begin{array}{l}\text { DBSCAN- } \\
\text { SWA (2020) }\end{array}$ & 2e61b95 & & $\begin{array}{l}\text { Numpy, Biopython, } \\
\text { sklearn, Prokka }\end{array}$ & $2.2 \mathrm{~GB}$ & $\begin{array}{l}\text { Gene } \\
\text { metrics, } \\
\text { alignments }\end{array}$ & $\begin{array}{l}\text { (Gan et al., } \\
\text { 2020) }\end{array}$ \\
\hline $\begin{array}{l}\text { VIBRANT } \\
(2020)\end{array}$ & 1.2 .1 & conda & $\begin{array}{l}\text { Python3, Prodigal, } \\
\text { HMMER3, BioPython, } \\
\text { Pandas, Matplotlib, } \\
\text { Seaborn, Numpy, } \\
\text { Scikit-learn, Pickle }\end{array}$ & $11 \mathrm{~GB}$ & $\begin{array}{l}\text { HMMs } \\
\text { (KEGG, Pfam, } \\
\text { VOG), } \\
\text { machine } \\
\text { learning }\end{array}$ & $\begin{array}{l}\text { (Kieft et al., } \\
\text { 2020) }\end{array}$ \\
\hline $\begin{array}{l}\text { PhageBoost } \\
\text { (2021) }\end{array}$ & 0.1 .7 & pip & Python3 & $13 \mathrm{MB}$ & $\begin{array}{l}\text { Gene and } \\
\text { nucleotide } \\
\text { metrics, } \\
\text { machine } \\
\text { learning }\end{array}$ & $\begin{array}{l}\text { (Sirén et al., } \\
\text { 2021) }\end{array}$ \\
\hline $\begin{array}{l}\text { VirSorter2 } \\
\text { (2021) }\end{array}$ & 2.2.1 & conda & $\begin{array}{l}\text { Python3, snakemake, } \\
\text { scikit-learn, } \\
\text { imbalanced-learn, } \\
\text { pandas, seaborn, } \\
\text { hmmer, prodigal, } \\
\text { screed }\end{array}$ & $12 \mathrm{~GB}$ & $\begin{array}{l}\text { Alignments, } \\
\text { HMMs }\end{array}$ & $\begin{array}{l}\text { (Guo et al., } \\
\text { 2021) }\end{array}$ \\
\hline
\end{tabular}

exact locations of the attachment site core duplications ( $a t t L$ and $a t t R)$ ? The runtime and CPU time in seconds, peak memory usage and file write operations were captured by Snakemake (Snakemake, RRID:SCR_003475) for the steps running the prophage tools only (not for any file conversion steps before or after running each tool). The predictions were then compared to the gold standard annotations and the number of true positive (TP), true negative (TN), false positive (FP) and false negative (FN) gene labels were used to calculate the performance metrics. Each application marks prophages slightly differently, and therefore we used the designation of coding sequence (CDS) features as phage or not to assess prophage predictions.

\section{Adding new genomes}

We developed the framework to simplify the addition of new genomes to the benchmarks. Each genome is provided in the standard GenBank format, and the prophages are marked by the inclusion of a non-standard flag for each genomic feature that indicates that it is part of a prophage. We use the qualifier/is_phage $=$ " 1 " to indicate prophage regions. 


\begin{tabular}{|c|c|}
\hline $\begin{array}{l}\text { Accuracy was calculated as the ratio of correctly labelled genes to all CDS features } \\
\text { from the GenBank file }\end{array}$ & $\frac{T P+T N}{T P+T N+F P+F N}$ \\
\hline $\begin{array}{l}\text { Precision was calculated as the ratio of correctly labelled phage CDS features to all predicted } \\
\text { prophage CDS features }\end{array}$ & $\frac{T P}{T P+F P}$ \\
\hline The $f_{1}$ Score was calculated as the harmonic mean of Precision and Recall & $2 \times \frac{(\text { Recall } \times \text { Precision })}{(\text { Recall }+ \text { Precision })}$ \\
\hline \multicolumn{2}{|c|}{$\begin{array}{l}\text { Accuracy provides an overall impression of correctness but is distorted by the vast difference in the numbers of } \\
\text { prophage and non-prophage CDS features present in the genomes. The current gold-standard set includes } 7,729 \\
\text { prophage proteins and } 177,649 \text { non-prophage proteins. Therefore, predicting everything as not coming from a } \\
\text { prophage will result in an accuracy of } 0.96 \text {. Similarly, identifying everything as coming from a prophage will result } \\
\text { in high Recall, since that favours minimising false negatives. In contrast, Precision favours minimising false-positives } \\
\text { and so only predicting very confident regions will result in high precision. The } f_{1} \text { Score is the most suitable for } \\
\text { comparing predictions as it gives equal weighting to both precision and recall, and thus balances the unevenness } \\
\text { inherent in this data. }\end{array}$} \\
\hline
\end{tabular}

\section{Results and discussion}

Software compared

We compared the availability, installation, and results from ten different prophage prediction algorithms (Table 1). Two-ProphET (Reis-Cunha et al., 2019) and LysoPhD (Niu et al., 2019)—could not be successfully installed and were not included in the current framework (see below). The remaining eight PhiSpy (Akhter et al., 2012), Phage Finder (Fouts, 2006), VIBRANT (Kieft et al., 2020), VirSorter (Roux et al., 2015), Virsorter2 (Guo et al., 2021), Phigaro (Starikova et al., 2020), PhageBoost (Sirén et al., 2021), and DBSCAN-SWA (Gan et al., 2020) were each used to predict the prophages in 49 different manually curated microbial genomes.

Most of these programs utilize protein sequence similarity and HMM searches of core prophage genes to identify prophage regions. PhageBoost leverages a large range of protein features (such as dipeptide and tripeptide combinations) with a trained prediction model. PhiSpy was originally designed to identify prophage regions based upon seven distinct characteristics: protein length, transcript directionality, AT and GC skew, unique phage words, phage insertion points, optionally phage protein similarity and sequence similarity. DBSCAN-SWA likewise uses a range of gene metrics and trained prediction models to identify prophages.

Regardless of whether annotations are available, Virsorter2, Phigaro, and PhageBoost all perform de novo gene prediction with Prodigal (Hyatt et al., 2010) and VirSorter uses MetaGeneAnnotator (Noguchi et al., 2008) for the same purpose. VIBRANT can take proteins if they have 'Prodigal format definition lines' but otherwise performs predictions with Prodigal. PhageBoost can take existing annotations but this requires additional coding by the user. DBSCAN-SWA can take annotations or can perform gene predictions with Prokka (Seemann, 2014). PhiSpy takes an annotated genome in GenBank format and uses the annotations provided.

\section{Ease of installation}

The prophage prediction packages Phigaro, PhiSpy, VIBRANT, VirSorter, and VirSorter2 are all able to be installed with conda from the Bioconda channel (Grüning et al., 2018), while Phispy, Phigaro, and PhageBoost can be installed with pipthe Python package installer. Phigaro, VIBRANT, VirSorter, and VirSorter2 require a manual one-time setup to download their respective databases. Phigaro uses hard-coded file paths for its database installation, either to the user's home directory or to a system directory requiring root permissions. Neither option is ideal as it is impossible to have isolated versions or installations of the program, and it prevents updating the installation paths of its dependencies. For PhageBoost to be able to take existing annotations, a custom script was created to skip the gene prediction stage and run the program. Basic PhiSpy functionality is provided without requiring third-party databases. However, if the HMM search option is invoked, a database of phage-like proteins - e.g. pVOG (Grazziotin et al., 2017), VOGdb (https://vogdb.org), or PHROGS (Terzian P et al., 2021) — must be manually downloaded before it can be included in PhiSpy predictions. DBSCAN-SWA is not currently available on any package manager and must be pulled from GitHub, however all its dependencies are available via conda and it could easily be added in the future. All the above "manual" installation and setup steps are uncomplicated and are automatically executed by the Snakemake pipelines provided in the framework. 
Phage Finder was last updated in 2006 and is not available on any package manager that we are aware of. The installation process is dated with the package scripts liberally utilising hard-coded file paths. The Snakemake pipeline for this package resolves this with soft links between the framework's directory to the user's home directory (where the package expects to be installed). The dependencies are available via conda allowing the complete installation and setup to be handled automatically by Snakemake.

LysoPhD does not appear to be available to download anywhere and was dropped from the comparison. ProphET requires the unsupported BLAST legacy and EMBOSS packages. It is not available on any package manager and instructions for a clean installation are incomplete and not compatible with conda. The codebase was last updated in 2019. Numerous issues were encountered installing dependencies and despite significant effort we were not able to create a working installation. ProphET's installation script reported many errors during setup, but alarmingly finished with an exit code zero to indicate a successful installation. Preparing the necessary GFF files in a format that program could use was non-trivial. The program reported errors during runtime that we believe are related to the errors encountered during installation; ProphET terminated with incomplete output but again returned an exit code zero to indicate a successful run. ProphET was dropped from the comparison.

\section{Prophage prediction performance}

There was minimal difference in the performance metrics for the different methods of running PhiSpy, and we have recently shown (Roach et al in preparation) that including HMM searches with PhiSpy results in less than one additional prophage being identified. Therefore, only PhiSpy using default settings will be discussed in comparison to the other tools. PhiSpy, VIBRANT, and Phigaro performed best for mean accuracy (Figure 1a; Table S3) while DBSCAN-SWA performed the worst. PhiSpy, Phigaro, and Phage Finder performed best for mean precision (Figure 1b; Table S3).
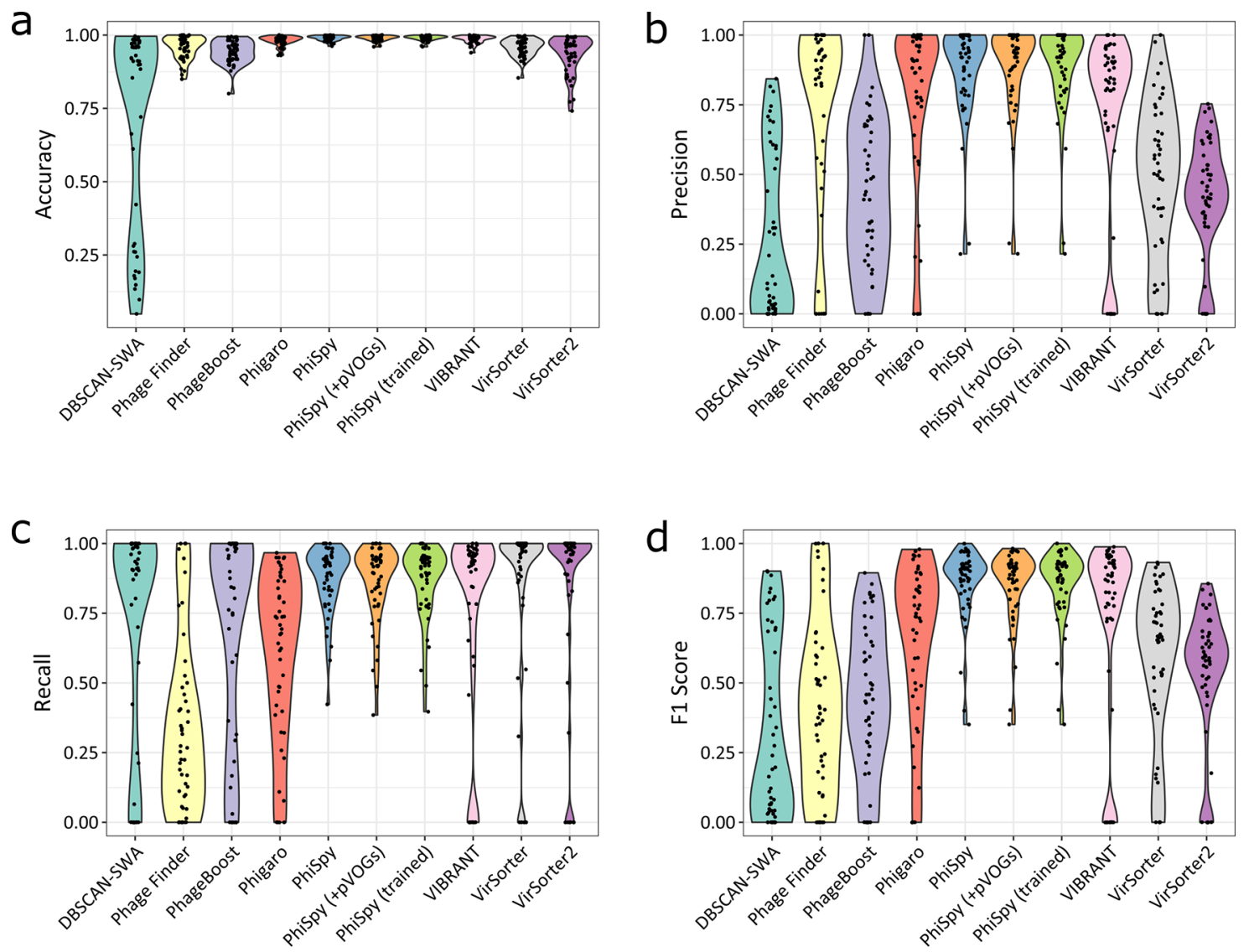

Figure 1. Prediction performance metrics for prophage callers. Violin plots for each tool are shown with individual points for each genome indicated. The graphs show: 'Accuracy' $(a)$ as the ratio of correctly labelled genes to all genes, 'Precision' $(b)$ as the ratio of correctly labelled phage genes to all predicted phage genes, 'Recall' $(c)$ as the ratio of correctly labelled phage genes to all known phage genes, and ' $f_{1} S c_{c o r e}(d)$ as defined in the methods. For all graphs, more is generally better. 
DBSCAN-SWA, PhageBoost, VirSorter, and VirSorter2 all performed poorly for mean precision. This was mostly driven by a high false-positive rate compared to the other tools (Figure S1). PhiSpy, VirSorter, VirSorter2, VIBRANT, DBSCAN-SWA, and PhageBoost all had high mean recall scores.

Each tool balances between recall and precision. For example, the more conservative Phage Finder performed relatively well in terms of precision, making very confident predictions, but had one of the lower mean recall ratios and was not predicting prophages based on limited information. In contrast, the more speculative DBSCAN-SWA and PhageBoost both exhibited the opposite trend.

The $\mathrm{f}_{1}$ Score is a more nuanced metric, as it requires high performance in both precision and recall. PhiSpy, VIBRANT, Phigaro, VirSorter, and VirSorter2 all averaged above 0.5, while the remaining tools suffered from too many false predictions (FP or FN) (Figure 1d).

\section{Runtime performance}

Many users will not be too concerned about runtime performance, for instance if they are performing a one-off analysis on a genome of interest all the tools will finish in a reasonable time. However, efficient resource utilization is an important consideration for large-scale analyses. Provisioning computing resources costs money and a well optimised tool that runs fast translates to real-world savings. The runtime distributions across the genomes are shown for each tool in Figure $2 \mathrm{a}$. The slowest prophage predictors were generally VirSorter and VirSorter2 with mean runtimes of 1,316 and 2,118 seconds respectively, except for a single DBSCAN-SWA run taking 4,697 seconds. PhiSpy using the trained datasets was by far the fastest performing tool (8.4 seconds mean runtime), although if an appropriate training set is not available for the genus of interest it would first need to be generated to benefit from these reduced runtimes. PhageBoost was the next
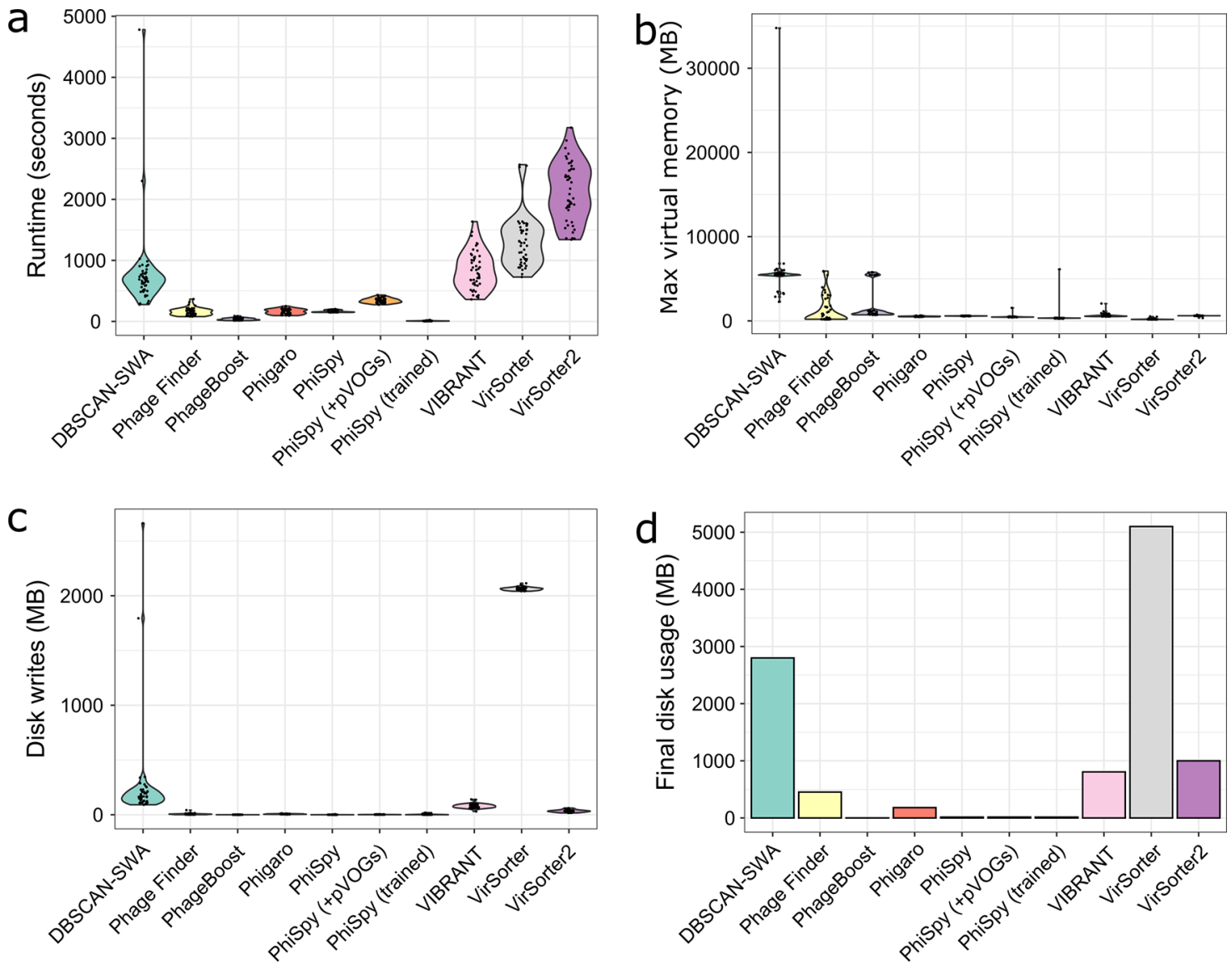

Figure 2. Runtime and peak memory usage comparison. Violin plots for each tool are shown with individual points for each genome indicated. The graphs show total runtime in seconds $(a)$, peak memory usage in MB $(b)$, total file writes in MB (c) and the final total disk usage (all genomes) in MB (d). For all graphs, less is better. 
fastest (37.8 seconds mean runtime) and Phage Finder, Phigaro, and PhiSpy with default parameters all performed similarly well in terms of runtime.

Memory requirements also remain an important consideration for provisioning resources for large-scale analyses. For instance, inefficiency is encountered where the memory required by single-threaded processes exceeds the available memory per CPU. Peak memory usage for each tool is shown in Figure 2b. Memory requirements were lowest for VirSorter and trained PhiSpy with 210 and $450 \mathrm{MB}$ mean peak memory respectively. There was a single notable exception for trained PhiSpy (predicting prophages in E. coli O157:57 EDL933) with a peak memory usage of 6.13 GB. DBSCAN-SWA had the highest mean peak memory of $6.0 \mathrm{~GB}$ with one run requiring $35 \mathrm{~GB}$ at its peak. Apart from the DBSCAN-SWA outlier, there were no situations where the peak memory usage would prevent the analysis from completing on a modest personal computer, but at larger-scales, Phigaro, PhiSpy, VirSorter, and VirSorter2 have an advantage in terms of peak memory usage.

Another important consideration for large-scale analyses are the file sizes that are generated by the different tools. Large output file sizes can place considerable strain on storage capacities, and large numbers of read and write operations can severely impact the performance of a system or HPC cluster for all users. Total file writes for the default files (in MB, including temporary files) are shown in Figure 2c and the final disk usage for all genomes for each tool is shown in Figure 2d. VirSorter, DBSCAN-SWA, and VirSorter2 performed the most write operations with mean file writes of $2.063,0.262$, and $0.034 \mathrm{~GB}$ respectively. The other tools performed similarly well and have a clear advantage at scale as they perform far fewer disk writes. VirSorter and DBSCAN-SWA removed most of their generated files, however, the final disk usage for these tools were still the highest at 5.36 and 2.96 GB respectively. Disk usage for PhageBoost and PhiSpy was by far the lowest at 0.14 and $15 \mathrm{MB}$ respectively.

\section{Caveats}

Every bioinformatics comparison involves many biases. In this comparison, PhiSpy performs well, but we developed PhiSpy and many of the gold-standard genomes were extensively used during its development to optimize the algorithm. VirSorter and VirSorter2 were primarily developed to identify viral regions in metagenomes rather than prophages in bacterial genomes — although they have been used for that e.g. in Glickman et al. (2020)—and filtering VirSorter and VirSorter2 hits with CheckV (Nayfach et al., 2021) is recommended. By openly providing the Prophage Prediction Comparison framework, creating a framework to install and test different software, and defining a straightforward approach to labelling prophages in GenBank files, we hope to expand our gold-standard set of genomes and mitigate many of our biases. We welcome the addition of other genomes (especially from beyond the Proteobacteria/Bacteroidetes/Firmicutes that are overrepresented in our gold-standard database).

Recent developments in alternative approaches to predict prophages, including mining phage-like genes from metagenomes and then mapping them to complete genomes (Nayfach et al., 2021) and using short-read mapping to predict prophage regions from complete bacterial genomes (Kieft \& Anantharaman, 2021) have the potential to generate many more ground-truth prophage observations. However, both approaches are limited as they will identify prophages that are active, but are unable to identify quiescent prophage regions, and thus for prophage prediction algorithms they will provide useful true positive datasets but may not provide accurate true negative datasets.

\section{Conclusions}

In this comparison, PhiSpy, VIBRANT, and Phigaro were the best performing prophage prediction tools for $\mathrm{f}_{1}$ score. PhiSpy and Phigaro were also among the best in terms of runtime performance metrics. Phage Finder performs well in terms of precision at the expense of false-negatives, whereas VirSorter, VirSorter2, DBSCAN-SWA and PhageBoost perform well for recall at the expense of false-positives. Currently, DBSCAN-SWA, VirSorter, and VirSorter2 are not as well suited for large-scale identification of prophages from complete bacterial genomes when compared to the other tools. More genomes with manually curated prophage annotations are needed, and we anticipate that these benchmarks will change with the addition of new genomes, the addition of new tools, and as the tools are updated over time. Developers are strongly encouraged to contribute by adding or updating their tool and adding their manually curated genomes to be included in the benchmarking. Users are strongly encouraged to check the GitHub repository for the latest results before making any decisions on which prophage prediction tool would best suit their needs.

\section{Author contributions}

RAE conceived of the study; KM and PD generated the initial gold-standard set and SKG, LI, and EP contributed to the gold-standard set; RAE and MJR created the framework; RAE, MJR, and SR performed the analysis. All authors contributed to the manuscript writing. 


\section{Data availability}

Underlying data

Zenodo: linsalrob/ProphagePredictionComparisons: Review release. https://doi.org/10.5281/zenodo.4739878. (Roach and Edwards, 2021b).

This project contains the following underlying data;

- genbank/

${ }^{\circ}$ The gold-standard prophage-annotated genomes in genbank format

- snakefiles/

o Snakemake pipeline files for running each of the prophage prediction tools against the gold-standard prophage-annotated genomes

- rules/

- Snakemake files with generic rules used by one or more of the Snakemake pipelines

- conda_environments/

${ }^{\circ}$ Configuration files for creating conda environments for use in the Snakemake pipelines

- data/

- Any custom small datasets required by the prophage prediction tools

- scripts/

${ }^{\circ}$ Perl and Python scripts that are used in the Snakemake pipelines for performing various tasks

- ProphagePredictionsLib/

${ }^{\circ}$ Library files required by the Perl and Python scripts

- jupyter_notebooks/

- Summary metric tables for all of the tools, and example Jupyter notebook for producing the comparison figures

- img/

${ }^{\circ}$ Example figures generated by the Jupyter notebook

\section{- LICENCE}

${ }^{\circ}$ Licence file for the github repository

- Supplementary/

${ }^{\circ}$ SupplementaryTables.xlsx

- (Sheet 1) Table S1. Genomes provided in the gold-standard library with manually curated prophages

- (Sheet 2) Table S2. Prophages identified in the genomes

- (Sheet 3) Table S3. Mean metrics for each tool as measured from our gold-standard set of genomes 
${ }^{\circ}$ FigureS1.tif

- False positive comparison.

Underlying data is also available at:

Github: Comparisons of multiple different prophage predictions https://github.com/linsalrob/ProphagePredictionComparisons/tree/v0.1-beta (Roach and Edwards, 2021a).

Extended data

Zenodo: Extended data for 'Philympics 2021: Prophage Predictions Perplex Programs': https://doi.org/10.5281/ zenodo.4739878.

This project contains the following extended data:

\section{SupplementaryTables.xlsx:}

- Table S1. Genomes provided in the gold-standard library with manually curated prophages

- Table S2. Prophages identified in the genomes

- Table S3. Mean metrics for each tool as measured from our gold-standard set of genomes

\section{FigureS1.tif:}

- Figure S1. False positive comparison. Violin plots for each tool show 'False Positives' as the number of genes incorrectly labelled prophage genes in each genome. Less is better.

Akhter S, Aziz RK, Edwards RA: PhiSpy: a novel algorithm for finding prophages in bacterial genomes that combines similarity- and composition-based strategies. Nucleic Acids Res. 2012; 40: e126-e126. PubMed Abstract | Publisher Full Text | Free Full Text

Amgarten D, Braga LPP, Da Silva AM, et al.: MARVEL, a Tool for Prediction of Bacteriophage Sequences in Metagenomic Bins. Front Genet. 2018; 9. PubMed Abstract | Publisher Full Text | Free Full Text

Arndt D, Grant JR, Marcu A, et al.: PHASTER: a better, faster version of the PHAST phage search tool. Nucleic Acids Res. 2016; 44: W16-W21. PubMed Abstract | Publisher Full Text| Free Full Text

Aziz RK, Bartels D, Best AA, et al.: The RAST Server: Rapid Annotations using Subsystems Technology. BMC Genomics. 2008; 9: 75.

PubMed Abstract | Publisher Full Text | Free Full Text

Breitbart M: Marine Viruses: Truth or Dare. Ann Rev Mar Sci. 2012; 4: 425-448.

PubMed Abstract | Publisher Full Text

Brüssow H, Canchaya C, Hardt W-D: Phages and the Evolution of Bacterial Pathogens: from Genomic Rearrangements to Lysogenic Conversion. Microbiol Mol Biol Rev. 2004; 68: 560-602.

PubMed Abstract | Publisher Full Text | Free Full Text

Calendar R: The Bacteriophages. New York, Springer US: Plenum Press; 1988.

Campbell AM: Preferential Orientation Preferential Orientation of Natural Lambdoid Prophages and Bacterial Chromosome Organization. Theor Popul Biol. 2002; 61: 503-507.

PubMed Abstract | Publisher Full Text

Canchaya C, Proux C, Fournous G, et al.: Prophage Genomics. Microbiol Mol Biol Rev. 2003; 67: 238-276.

PubMed Abstract | Publisher Full Text | Free Full Text
Casjens S: Prophages and bacterial genomics: what have we learned so far? Mol Microbiol. 2003; 49: 277-300. PubMed Abstract | Publisher Full Text

Dutilh BE, Cassman N, Mcnair K, et al.: A highly abundant bacteriophage discovered in the unknown sequences of human faecal metagenomes. Nat Commun. 2014; 5: 4498. PubMed Abstract | Publisher Full Text | Free Full Text

Fouts DE: Phage_Finder: Automated identification and classification of prophage regions in complete bacterial genome sequences. Nucleic Acids Res. 2006; 34: 5839-5851.

PubMed Abstract | Publisher Full Text | Free Full Text

Gan R, Zhou F, Si Y, et al.: DBSCAN-SWA: an integrated tool for rapid prophage detection and annotation. bioRxiv. 2020; 2020.07.12.199018. Publisher Full Text

Glickman C, Kammlade SM, Hasan NA, et al.: Characterization of integrated prophages within diverse species of clinica nontuberculous mycobacteria. Virol J. 2020; 17: 124. PubMed Abstract | Publisher Full Text | Free Full Text Grazziotin AL, Koonin EV, Kristensen DM: Prokaryotic Virus Orthologous Groups (pVOGs): a resource for comparative genomics and protein family annotation. Nucleic acids res. 2017; 45: D491-D498. PubMed Abstract | Publisher Full Text | Free Full Text

Grigoriev A: Analyzing genomes with cumulative skew diagrams. Nucleic Acids Res. 1998; 26: 2286-2290.

PubMed Abstract | Publisher Full Text | Free Full Text

Grüning B, Dale R, Sjödin A, et al.: Bioconda: sustainable and comprehensive software distribution for the life sciences. Nat Methods. 2018; 15: 475-476.

PubMed Abstract | Publisher Full Text 
Guo J, Bolduc B, Zayed AA, et al.: VirSorter2: a multi-classifier, expertguided approach to detect diverse DNA and RNA viruses. Microbiome. 2021; 9: 37.

PubMed Abstract | Publisher Full Text | Free Full Text

Hyatt D, Chen G-L, Locascio PF, et al.: Prodigal: prokaryotic gene recognition and translation initiation site identification. $B M C$ bioinformatics. 2010; 11: 119-119.

PubMed Abstract | Publisher Full Text | Free Full Text

Kang HS, Mcnair K, Cuevas DA, et al.: Prophage genomics reveals patterns in phage genome organization and replication. bioRxiv. 2017: 114819.

Publisher Full Text

Kieft K, Anantharaman K: Deciphering active prophages from metagenomes. bioRxiv. 2021: 2021.01.29.428894.

Publisher Full Text

Kieft K, Zhou Z, Anantharaman K: VIBRANT: automated recovery, annotation and curation of microbial viruses, and evaluation of viral community function from genomic sequences. Microbiome. 2020; 8: 90. PubMed Abstract | Publisher Full Text | Free Full Text

Köster J, Rahmann S: Snakemake-a scalable bioinformatics workflow engine. Bioinformatics. 2012; 28: 2520-2522.

PubMed Abstract | Publisher Full Text

Lima-Mendez G, Van Helden J, Toussaint A, et al.: Prophinder: a

computational tool for prophage prediction in prokaryotic genomes. Bioinformatics. 2008; 24: 863-865.

PubMed Abstract| Publisher Full Text

Mcnair K, Zhou C, Dinsdale EA, et al.: PHANOTATE: a novel approach to gene identification in phage genomes. Bioinformatics. 2019; 35 4537-4542.

PubMed Abstract | Publisher Full Text | Free Full Text

Nayfach S, Camargo AP, Schulz F, et al.: CheckV assesses the quality and completeness of metagenome-assembled viral genomes. Nat Biotechnol. 2021; 39: 578-585.

PubMed Abstract | Publisher Full Text | Free Full Text

Niu Q, Peng S, Zhang X, et al.: LysoPhD: predicting functional prophages in bacterial genomes from high-throughput sequencing. 2019 IEEE International Conference on Bioinformatics and Biomedicine (BIBM), 18-21

Nov. 2019. 2019; 1-5.

Publisher Full Text
Noguchi $\mathrm{H}$, Taniguchi $\mathrm{T}$, Itoh T: MetaGeneAnnotator: detecting speciesspecific patterns of ribosomal binding site for precise gene prediction in anonymous prokaryotic and phage genomes. DNA res. 2008; 15: 387-396. PubMed Abstract | Publisher Full Text | Free Full Text

Reis-Cunha JL, Bartholomeu DC, Manson AL, et al.: ProphET, prophage estimation tool: A stand-alone prophage sequence prediction tool with self-updating reference database. PLOS ONE. 2019; 14: e0223364. PubMed Abstract | Publisher Full Text | Free Full Text

Roach MJ, Edwards RA: linsalrob/ProphagePredictionComparisons [Online]. GitHub. 2021a. [Accessed].

Reference Source

Roach MJ, Edwards RA: linsalrob/ProphagePredictionComparisons: Review release (Version v0.1). Zenodo. 2021b.

Roux S, Enault F, Hurwitz BL, et al.: VirSorter: mining viral signal from microbial genomic data. PeerJ. 2015; 3: e985.

PubMed Abstract | Publisher Full Text | Free Full Text

Seemann T: Prokka: rapid prokaryotic genome annotation.

Bioinformatics. 2014; 30: 2068-2069.

PubMed Abstract | Publisher Full Text

Sirén K, Millard A, Petersen B, et al.: Rapid discovery of novel prophages using biological feature engineering and machine learning. NAR

Genom Bioinform. 2021; 3 .

Publisher Full Text

Song W, Sun H-X, Zhang C, et al.: Prophage Hunter: an integrative hunting tool for active prophages. Nucleic Acids Res. 2019; 47: W74-W80. PubMed Abstract | Publisher Full Text | Free Full Text

Sousa ALD, Maués D, Lobato A, et al.: PhageWeb - Web Interface for Rapid Identification and Characterization of Prophages in Bacterial Genomes. Fron Genet. 2018; 9.

PubMed Abstract | Publisher Full Text | Free Full Text

Starikova EV, Tikhonova PO, Prianichnikov NA, et al.: Phigaro: highthroughput prophage sequence annotation. Bioinformatics. 2020; 36 3882-3884.

PubMed Abstract | Publisher Full Text

Terzian P, Olo Ndela E, Galiez C, et al.: PHROG: families of prokaryotic virus proteins clustered using remote homology. [Online]. 2021.

[Accessed June 2021].

Reference Source 


\section{Open Peer Review}

\section{Current Peer Review Status: ? $\mathrm{X}$}

\section{Version 1}

Reviewer Report 31 August 2021

https://doi.org/10.5256/f1000research.57937.r91781

(C) 2021 Anantharaman $\mathrm{K}$ et al. This is an open access peer review report distributed under the terms of the Creative Commons Attribution License, which permits unrestricted use, distribution, and reproduction in any medium, provided the original work is properly cited.

\section{Karthik Anantharaman}

Department of Bacteriology, University of Wisconsin-Madison, Madison, WI, USA

\section{Kristopher Kieft}

Department of Bacteriology, University of Wisconsin-Madison, Madison, WI, USA

This manuscript by Roach et al. describes the creation and benchmarking of a gold standard bacterial dataset that can be used for prediction of prophages from bacterial genomes.

Additionally, they also benchmark seven currently available software for prediction of prophages.

Overall, this manuscript and the datasets provided represent a valuable resource to the community that can be used widely. We do have some comments that in our opinion can improve the manuscript, the benchmarking and the utility of the gold standard dataset that the authors aim to to provide to the community.

\section{Introduction:}

Last paragraph: the reasoning for this study is sound and accurate. Though the usage of the term "annotated" is slightly ambiguous here, and throughout the paper, because it is meant to designate either prophage locations or protein/gene annotations.

\section{Methods:}

First sentence: the actual method of "manually curated prophage annotations was generated" is never explained.

What method was used? How were prophages manually identified, annotated, and validated? To me, gold standard would imply each prophage has been experimentally validated.

Was there is specific method by which certain hosts were selected? I can see that there is "Escherichia_coli_O157-H7" and "Escherichia_coli_O157-H7_EDL933". Why were so similar hosts chosen? Do they have significantly different prophages? There are multiple examples of this. 
Many also appear to be common model organisms. How was diversity ensured? Is there a possibility to use organisms more widely distributed across the tree of life?

If this component is at all incorrect then the performance metrics, especially false positives if the prophage boundaries are wrong, will be biased.

How was Supplemental Table 2 generated?

Overall, there is no indication as to how the gold standard dataset, the centerpiece of the paper, was actually generated. This does not significantly affect the study's results, but this information needs to be included before publication. For example, were the host sequences chosen at random from a database? Represent variable phylogenetic backgrounds? Source environments? Was prophage phylogeny taken into account? There are certainly questions left unanswered.

\section{Results:}

Prophage prediction performance: rather than only providing subjective "best" and "worst" designations, the numerical results should be provided too (e.g., accuracy of $0.9+/-0.1$ ). Furthermore, what statistical metrics were used to designate "best" and "worst"?

Caveats: The VIBRANT manuscript says that it also was developed to identify both temperate and virulent viral regions in metagenomes. FYI - we were not able to access the Supplementary datasets through the paper (but were able to retrieve them from bioRxiv).

\section{Suggestions:}

Overall, this comparison framework would benefit from simple, minor additions.

Does the specific host (e.g., taxonomy) affect the results of prophage prediction for some tools? For tools that utilize HMM searches, the HMM databases may be biased towards certain groups (e.g., E. coli phages). We suggest that a comparison of precision/recall be compared to the source host.

Do the performance comparisons only take into account total prophage CDS predictions or also the completeness of predicted prophages? For example, identifying all prophages but only $50 \%$ of each of those prophages is different than identifying half of all prophages but $100 \%$ of each of those.

Is there any effect on hosts with multiple prophages? Are some tools affected by this?

Some tools will have predicted prophages that were not in the gold standard set (false positives). What measures were taken to ensure that none of these are real prophages that were missed within the manual curation?

Is the work clearly and accurately presented and does it cite the current literature? Yes

Is the study design appropriate and is the work technically sound? 
Partly

Are sufficient details of methods and analysis provided to allow replication by others? Partly

If applicable, is the statistical analysis and its interpretation appropriate?

Yes

Are all the source data underlying the results available to ensure full reproducibility?

Yes

Are the conclusions drawn adequately supported by the results?

Yes

Competing Interests: No competing interests were disclosed.

Reviewer Expertise: Metagenomics, Microbial and Phage ecology

We confirm that we have read this submission and believe that we have an appropriate level of expertise to state that we do not consider it to be of an acceptable scientific standard, for reasons outlined above.

Author Response 05 Apr 2022

Michael Roach

Thank you so much for reviewing our manuscript. We hope that version 2 address the concerns with the manuscript which we outline below:

Last paragraph: the reasoning for this study is sound and accurate. Though the usage of the term "annotated" is slightly ambiguous here, and throughout the paper, because it is meant to designate either prophage locations or protein/gene annotations.

Response: We agree that this could be clearer. We have updated all instances of 'annotations' in the manuscript to explicitly refer to either prophage or gene annotations.

Methods: First sentence: the actual method of "manually curated prophage annotations was generated" is never explained. What method was used? How were prophages manually identified, annotated, and validated? To me, gold standard would imply each prophage has been experimentally validated.

Response: Experimentally validating all prohages by inducing them is not possible currently, as we do not know the signal for triggering the lytic cycles for all the prophages we know about. It is also not possible for elements such as cryptic phages, which may still offer a strong phrophage signal and represent true phage genome sequence, as they are not able to be induced. For cryptic prophages we believe it is still important to include these predictions as prophage annotations. The next best approach to a gold-standard library is to manually inspect and annotate the genomes. We have included the guidelines we use as supplementary material. 
Was there is specific method by which certain hosts were selected? I can see that there is "Escherichia_coli_O157-H7" and "Escherichia_coli_O157-H7_EDL933". Why were so similar hosts chosen? Do they have significantly different prophages? There are multiple examples of this. Many also appear to be common model organisms. How was diversity ensured? Is there a possibility to use organisms more widely distributed across the tree of life?

Response: For PhiSpy's original development it was important to include multiple similar genomes with dissimilar numbers and positions of phages for training the algorithm. This selection bias is something that we have more recently been trying to address. We have earmarked many new diverse genomes for manual curation, and this update marks the inclusion of 10 new prophage annotated genomes from underrepresented phyla. There is still a long way to go. Annotating prophages remains an extremely challenging task for underrepresented bacterial phyla, and it will remain so until our knowledgebase of known phages and phage proteins for these phyla improves.

If this component is at all incorrect then the performance metrics, especially false positives if the prophage boundaries are wrong, will be biased.

Response: The current state is not perfect, rather, it is the best we can do right now. We agree with the sentiment, and it is why we have designed the repository around making it easy to add and refine tools, genomes and prophage annotations over time. However, we don't believe there are enough errors to significantly affect the outcome of the evaluation.

How was Supplemental Table 2 generated?

Response: It was originally compiled during manual curation. We now include a script for generating this table from the prophage-annotated GenBank files, as well as an updated table to include the new genomes.

Overall, there is no indication as to how the gold standard dataset, the centerpiece of the paper, was actually generated. This does not significantly affect the study's results, but this information needs to be included before publication. For example, were the host sequences chosen at random from a database? Represent variable phylogenetic backgrounds? Source environments? Was prophage phylogeny taken into account? There are certainly questions left unanswered.

Response: We hope the inclusion of our guidelines for manual curation and our recent progress has alleviated these concerns. There is still a long way to go to achieve a more diverse representation of prophages and this framework is intended to support this journey.

Results: Prophage prediction performance: rather than only providing subjective "best" and "worst" designations, the numerical results should be provided too (e.g., accuracy of $0.9+/-0.1)$. Furthermore, what statistical metrics were used to designate "best" and "worst"?

Response: This was partially available in Supp table 3, but given its importance, we have move this to a new table (Table 2 ) and include a new table (Table 3) for the benchmarking results. We have also calculated and report standard deviations where 
applicable. "Best" and "Worst" are simply designated based on the rankings of each prophage caller based on their scores for a given metric.

Caveats: The VIBRANT manuscript says that it also was developed to identify both temperate and virulent viral regions in metagenomes. FYI - we were not able to access the Supplementary datasets through the paper (but were able to retrieve them from bioRxiv). Response: Our apologies. The Genbank files are stored and can be retrieved using git-Ifs. The Zenodo repo is a verbatim copy of the GitHub repo and does not resolve the git-Ifs links to the GenBank files. We have added a section in underlying data to clarify this. We have also made sure there are instructions in the GitHub readme and genbank subfolder readme for syncing the GenBank files with git-lfs.

Suggestions: Overall, this comparison framework would benefit from simple, minor additions. Does the specific host (e.g., taxonomy) affect the results of prophage prediction for some tools? For tools that utilize HMM searches, the HMM databases may be biased towards certain groups (e.g., E. coli phages). We suggest that a comparison of precision/recall be compared to the source host.

Response: This is absolutely a consideration for every program and comes back to the problem of selection bias in the database. It's fundamentally not something we can solve in this update of the framework. We compared f1 scores for each tool against genus and the f1 scores of each genus across the population averages (see results and Figure $\mathrm{S} 2 \mathrm{a} / \mathrm{b}$ ).

Do the performance comparisons only take into account total prophage CDS predictions or also the completeness of predicted prophages? For example, identifying all prophages but only $50 \%$ of each of those prophages is different than identifying half of all prophages but $100 \%$ of each of those.

Response: The performance comparisons currently only compare the numbers of correctly labelled genes. If a user were manually curating their predictions, then it could be argued that partially capturing all prophages would be better than completely capturing half of the prophages. This is something we might be able to add in the future if there is enough interest.

Is there any effect on hosts with multiple prophages? Are some tools affected by this?

Response: The number of prophages shouldn't impact the performance of any of the tools but this is not something we've looked at specifically. Most genomes only have 1 or a few prophages and it would be difficult to draw any conclusions without more examples at the fringes ( 0 prophages or say more than 6 or so). It is certainly something to consider as the dataset grows.

Some tools will have predicted prophages that were not in the gold standard set (false positives). What measures were taken to ensure that none of these are real prophages that were missed within the manual curation?

Response: The genomes are small enough that the entire genomes are thoroughly examined during manual curation. We don't anticipate enough errors to significantly affect the outcome of the evaluation. Nevertheless, we do anticipate that some 
corrections will need to be made over time, be that from missed prophages or incorrect prophage boundaries and we welcome feedback from the community about the prophage annotations.

Competing Interests: The authors declare that there are no conflicts of interest.

Reviewer Report 23 August 2021

https://doi.org/10.5256/f1000research.57937.r91324

(C) 2021 Nobrega F. This is an open access peer review report distributed under the terms of the Creative Commons Attribution License, which permits unrestricted use, distribution, and reproduction in any medium, provided the original work is properly cited.

\section{Franklin Nobrega}

School of Biological Sciences, Faculty of Environmental and Life Sciences, University of Southampton, Southampton, UK

The manuscript by Roach and co-authors focuses on a crucial question on how to accurately determine prophage regions. With the increasing accessibility of HPC infrastructure by scholars, and the increasing number of open datasets available for mining, there is an urgent need to establish FAIR datasets to set a ground-truth for data analysis. This work sets the tone so the community can move away from using a favourite tool, to using the most accurate and reliable to address the question at hand. In particular, the tool performance part is excellently achieved, providing details for both the novice and the advanced user when considering a new tool for their pipeline. In sum, it was a pleasure to read this well-structured and well-balanced work, which I fully endorse. I would like to leave the authors with a few recommendations and suggestions.

\section{Recommendations:}

1. I believe the authors could use a more recent reference for Calendar, 1988 (Introduction).

2. Please provide the methods used for manual curation of prophage annotations, as this will contribute to increase the gold-standard genomes available.

3. What was the rationale for running PhiSpy using all modes, and not doing the same for other tools that also have different run modes? This would make for a more fair comparison.

4. Could the authors clarify which prophage prediction categories were considered for VirSorter and Virsorter2, as these will certainly affect the accuracy and recall results obtained. Similar comment for VIBRANT.

5. Data would benefit from statistical analysis.

\section{Suggestions:}


1. The first three lines of "Benchmark metrics" (Methods) seem to be more adequate to the Results section.

2. I would suggest that the authors summarize the information provided in "Software compared" (Results and discussion) as a supplementary table, since this will certainly be useful to the community.

Is the work clearly and accurately presented and does it cite the current literature? Yes

Is the study design appropriate and is the work technically sound?

Yes

Are sufficient details of methods and analysis provided to allow replication by others? Yes

If applicable, is the statistical analysis and its interpretation appropriate?

No

Are all the source data underlying the results available to ensure full reproducibility? Yes

Are the conclusions drawn adequately supported by the results? Yes

Competing Interests: No competing interests were disclosed.

Reviewer Expertise: Phage biology, phage-host interactions

I confirm that I have read this submission and believe that I have an appropriate level of expertise to confirm that it is of an acceptable scientific standard, however I have significant reservations, as outlined above.

Author Response 05 Apr 2022

Michael Roach

Thank you so much for reviewing our manuscript. We hope that version 2 addresses the concerns with the manuscript which we outline below:

I believe the authors could use a more recent reference for Calendar, 1988 (Introduction).

Response: We have updated this section with more recent references.

Please provide the methods used for manual curation of prophage annotations, as this will contribute to increase the gold-standard genomes available.

Response: Guidelines are now included in additional data.

What was the rationale for running Phispy using all modes, and not doing the same for 
other tools that also have different run modes? This would make for a more fair comparison.

Response: We developed PhiSpy and are well familiar with the different ways of running the pipeline. To keep the comparison fair, we now only present running Phispy with default parameters.

Could the authors clarify which prophage prediction categories were considered for VirSorter and Virsorter2, as these will certainly affect the accuracy and recall results obtained. Similar comment for VIBRANT.

Response: Following valuable contributions and input from SR-now a coauthor on this paper-the Virsorter categories 1-5 are taken as prophage predictions. Virsorter2 is run with --high-confidence-only --exclude-It2gene and we accept both predicted whole phages and integrated phage genomes as prophage predictions. VIBRANT is run with default parameters and we use all predictions from the integrated_prophage_coordinates output file.

Data would benefit from statistical analysis.

Response: We now include some statistical analysis as part of out database bias evaluation.

Suggestions: The first three lines of "Benchmark metrics" (Methods) seem to be more adequate to the Results section.

Response: We agree, the sentence has been moved to the results section.

I would suggest that the authors summarize the information provided in "Software compared" (Results and discussion) as a supplementary table, since this will certainly be useful to the community.

Response: We considered adding a more simplified summary table, such as ticks and crosses or 5-start ratings for various features, however these can be very subjective and would simply be our interpretation complete with our personal biases of the results.

Competing Interests: The authors declare that there are no conflicts of interest. 
The benefits of publishing with F1000Research:

- Your article is published within days, with no editorial bias

- You can publish traditional articles, null/negative results, case reports, data notes and more

- The peer review process is transparent and collaborative

- Your article is indexed in PubMed after passing peer review

- Dedicated customer support at every stage

For pre-submission enquiries, contact research@f1000.com 